\title{
CFD modeling and environmental assessment of a VOC removal silo
}

\author{
A. Egedy ${ }^{1}$ ○ $\cdot$ L. Gyurik ${ }^{1} \cdot$ Z. Ulbert ${ }^{1} \cdot$ A. Rado ${ }^{2}$
}

Received: 11 March 2020 / Revised: 27 May 2020 / Accepted: 30 June 2020 / Published online: 7 July 2020

(c) The Author(s) 2020

\begin{abstract}
Volatile organic compounds (VOC) are widely used in different industries as solvents or reagents. The removal of excess organic substances is vital from both environmental and economic point of view. The purge columns or silos where the solid particles are discharged continuously and the gas is fed counter currently are examples of operation units where the excess organic substances should be removed. In this study, a previously developed model of a VOC removal silo was used in the investigation of its extended operation. Both economic and environmental concerns were addressed. Response surface methodology (RSM) was applied for a two-factor environmental-economic optimization of the system utilizing temperature and purge gas flow rate as factors, and removal time and energy demand as outcomes. The environmental performance was tested using a simplified life cycle analysis (LCA). Two scenarios were examined and compared, one of them with full and the other one with zero VOC emission case. The main achievement of our study is a new optimization map which includes both environmental and economic aspects. In conclusion, $80{ }^{\circ} \mathrm{C}$ temperature and $3 \mathrm{~m}^{3} / \mathrm{h}$ flow rates resulted as the optimal parameters.
\end{abstract}

Keywords VOC removal · CFD simulation · Multi-scale model $\cdot$ LCA $\cdot$ Environmental calculation $\cdot$ Economic and environmental optimum

\section{Introduction}

Organic solvents are often present in various technologies, for example in pharmaceutical and chemical technologies, by polymerization or by resin production. However, the volatile organic compounds (VOC) are harmful substances, which need to be removed from the tail gas or waste fluids before being emitted to the environment (Zoveidavianpoor et al. 2012). The removal of VOC is an environmental issue, and it is our responsibility to preserve the biodiversity of our planet for future generations.

The VOC removal processes can be divided into subtypes: physical, chemical and biological separation (Berenjian et al. 2012). The VOC can be removed mostly by using adsorption processes where some adsorbent (for example,

Editorial responsibility: Samareh Mirkia.

A. Egedy

egedya@fmt.uni-pannon.hu

1 Department of Process Engineering, University of Pannonia, Egyetem St. 10, Veszprém 8200, Hungary

2 Hamburger Hungária Kft, Dunaújváros, Hungary activated carbon and zeolite) is used to extract the VOC from the gases. However, after a specific time, the adsorbent saturates with the VOC and needs to be purified. There are multiple ways to treat saturated adsorbents. The two most frequently used methods are incineration and regeneration (EPA-450 1983).

Incineration is considered to be the most harmful operation because the halogenated compounds like sulfur and nitrogen oxides might enter the atmosphere. For example, when activated carbon is incinerated, the $\mathrm{CO}_{2}$ produced is a primary contributor to the greenhouse effect. The incineration-based line suggests that the easiest way of treating harmful substances is combusting the VOC. The incineration of hydrocarbon-based substances also produces a large amount of $\mathrm{CO}_{2}$, in the case of which the application of catalysts can result in a lower environmental burden.

Regeneration has a much less negative impact on the environment. It occurs when one of the process variables is changed, e.g., temperature or pressure. Due to these different conditions, the VOC leaves the surface of the compounds, which results in a much lower concentration of harmful components, even if the gases are emitted into the atmosphere. 
Recovery-based technologies for VOC removal can either be wet or dry ones. Usually, when using a wet scrubber the efficiency can be improved by facilitating photocatalytic methods (similar to the Fenton process for nitrogen oxides removal). In the experimental research of Xie et al., ethyl acetate and toluene were used as volatile organic compounds to be removed in a bubble column (Xie et al. 2019). Peroxymonosulfate was used as an oxidizing agent in a semi-batch operation, and a maximum of $98.3 \%$ and $96.5 \%$ removal efficiencies were obtained for the ethyl acetate and toluene, respectively. The wet processes appear to be efficient alternatives to remove VOC; however, they are usually expensive. VOC adsorption can be an alternative way to the reactionbased system even in the case of multistage gas systems (Dobslaw et al. 2017).

There are several applications for the dry stripping as well. It is most often used when VOC needs to be removed from drinking water. This method can be carried out in packed column equipment, and a detailed mathematical model can help with the optimization and safe operation of the processes (Ghoreyshi et al. 2014). Another method is called purging. The main idea behind this is to use a countercurrent gas stream to remove the reagents or solvents from the surface of the feedstock. The position of the injection of the gas stream can be considered as a design variable, and its suitable selection can lead to an optimal operation range (Bobst et al. 1983). The purge vessels can be operated continuously in terms of both gas and solid phase. The discharge of the solid material and the phase contact can be optimized by the proper design of the conical part of the silo (or even by the implementation of inserts) (Wang et al. 2019).

VOC materials are harmful for human health as well as for the environment. The major factors contributing to this phenomenon are from industrial sources. Therefore, the VOC emission of factories should be maintained at the lowest possible levels. Smog formation is also an alerting problem, especially in the densely populated regions of China (Song et al. 2019), and its effects are the most severe in colder seasons (Yang et al. 2018). During the production of plastic products, granulates contact several VOC materials and solvent monomers.

Recently, recovery-based technologies are increasing in popularity. Unfortunately, both the wet and dry technologies have a disadvantage in the post-production processes, in which it is necessary to treat the purge gas (or waste fluids) produced. However, recovery-based technologies have a lower effect on the environment compared to the incineration processes. In our research, we attempt to model and simulate a purging process in which polymer granulates are dry-purged with nitrogen in a silo.
Silos are widely used equipment for storage, especially in food industries. The discharge of the silo is one of the primary processes to be treated, and the angle of the conical part plays a significant part in this operation. Funneling effect should be avoided because the homogenous discharge (mass flow) is preferable. This statement also applies for purge columns, where the average residence time of the particles should be around the same value or above the purging time. Besides the angle, the operation can be enhanced by utilizing different insert elements that are able to shift the flow patterns in the desired direction. Computational fluid dynamics (CFD) simulators can be used effectively to optimize the placement of insert elements inside the silo. However, in this case, it does not mean that we apply any optimization software or known optimization algorithms, because the long CFD simulation runtime makes the iterative optimization methods hard to use. The implementation of a model with the whole number of adsorbent particles is difficult, even on a laboratory scale.

However, most researches show that the multi-scale modeling approach facilitates a more effective outcome. Starting with a model validated for a single particle, we can obtain kinetic related information, then move on to the level of particle bulks which can result in a model providing the necessary information related to industrial scales (Zhou et al. 2013; Wehinger et al. 2015). The number of mesh elements in the CFD model of purification equipment can be increased to an enormous number in case of a multiparticle system, leading to an unstable solution, especially when some particle size modification processes (breaking, shrinking, leaching) take place within the system (Ström et al. 2015). Even so, adsorption (or desorption) can be calculated using multi-scale models (White 2012). All of the researches facilitate laboratory-scale models and try to extrapolate it to the industrial level.

In our previous study, we have investigated the possibility of using different spatial scales (2D and 3D) and multilevel models organized at different hierarchy levels (the particles' and the equipment's) for the modeling of the VOC removal process (Egedy et al. 2019). All the model types have been shown to be useful to a specific extent, and the information obtained from the lower hierarchy level can be transferred to the higher hierarchy level. This multilevel modeling approach can be used in the technology development processes.

In this study of ours, we decided to apply the response surface methodology (RSM), which is mostly used in the experimental design procedure. Following the work of Box and Wilson, we applied multilevel models using two or more factors and one or more outcomes (Box and Wilson 
Table 1 The experimental plan

\begin{tabular}{lrllllr}
\hline Experiment & $\begin{array}{l}\text { Tempera- } \\
\text { ture }\left[{ }^{\circ} \mathrm{C}\right]\end{array}$ & $\begin{array}{l}\text { Flow rate } \\
{\left[\mathrm{m}^{3} / \mathrm{h}\right]}\end{array}$ & $\begin{array}{l}\text { Factor 1 (scaled } \\
\text { temperature) }\end{array}$ & $\begin{array}{l}\text { Factor2 } \\
\text { (scaled flow } \\
\text { rate) }\end{array}$ & $\begin{array}{l}\text { Outcome1 } \\
\text { energy demand }\end{array}$ & $\begin{array}{l}\text { Outcome2 } \\
\text { removal time }\end{array}$ \\
\hline 1 & 60 & 3 & -1 & -1 & 68.7 & 108 \\
2 & 80 & 3 & 0 & -1 & 100.4 & 69 \\
3 & 100 & 3 & 1 & -1 & 135.6 & 46 \\
4 & 60 & 6 & -1 & -0.333 & 130.0 & 108 \\
5 & 80 & 6 & 0 & -0.333 & 190.4 & 69 \\
6 & 100 & 6 & 1 & -0.333 & 257.4 & 47 \\
7 & 60 & 12 & -1 & 1 & 273.2 & 109 \\
8 & 80 & 12 & 0 & 1 & 400.1 & 70 \\
9 & 100 & 12 & 1 & 1 & 541.7 & 47 \\
\hline
\end{tabular}

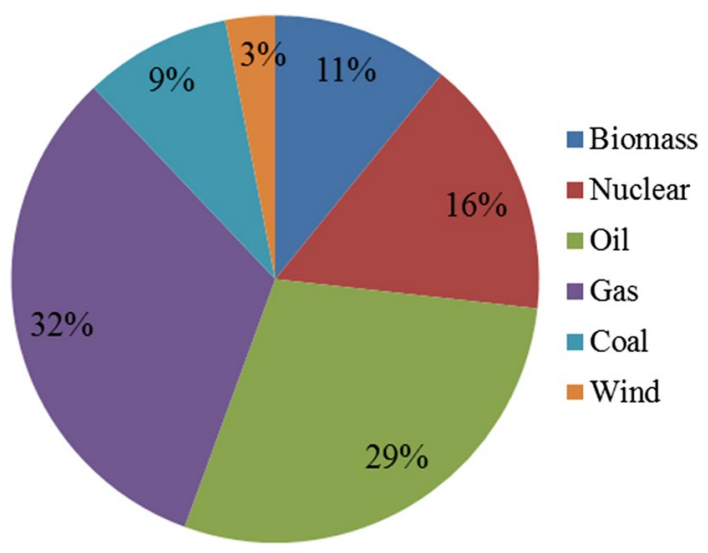

Fig. 1 The contributors to the Hungarian energy mix (Introduction to Hungarian Energy Market, 2018)

1951). Using this experimental design, the significant factors affecting the processes can be identified and the optimal process parameters can be estimated with lower computational demand. RSM is generally used in many research fields due to its ability of reducing the experimental time, the number of experiments and their costs. RSM was applied in researches related to $\mathrm{CO}_{2}$ capture (Hemmati et al. 2019), pyrolysis (Shalmashi and Khodadadi 2019), biodiesel synthesis (Jung et al. 2016), and even to shape optimization with CFD applications (Coppedè et al. 2019).

In addition, we have also examined the environmental effects of the VOC removal process. The environmental assessment can be based on several indicators. Oliva et al. developed indicators for a VOC removal process facilitating removal efficiency and environmental aspect as well (Oliva et al. 2018). In our case, we have decided to calculate a simplified life cycle assessment (LCA) for the VOC removal process using gate-to-gate type of analysis. Life cycle assessment or life cycle sustainability assessment (LCSA) has received much more attention in the last few decades as a comprehensive tool for evaluating different processes from an environmental point of view (Costa et al. 2019). This methodology can be generalized, and many databases can support the analysis. Furthermore, the methodology is applicable not only for conventional technologies but also for polymer recycling (Wang et al. 2019) or bioenergy production (Ubando et al. 2019) as well.

In our previous work, different spatial dimensions and scales (particle and laboratory scale) were tested (Egedy et al. 2019; Egedy and Rado 2018). In this study, a laboratory-scale silo was modeled. Temperature and purge gas flow rate were applied as factors, while removal time and energy need were used as outcomes. Two scenarios were compared during the LCA analysis: one with full VOC emission, and the other with its removal. Based on the RSM results, an optimal economic operating point was found, and based on the LCA analysis we found an optimal operating point in regard to environmental considerations. Then, we calculated an indicator number taking both the environmental and economic aspects of the technology into account, which resulted in the optimal operating point of the system. COMSOL Multiphysics software was used for the CFD simulations; MATLAB and R software packages were used to imply the RSM and the statistical calculations. Aspen Plus was used for the energy calculations, and OpenLCA 1.10 software (http:// www.openlca.org/) was applied for the LCA analysis. 
Fig. 2 a The applied geometry, b results of mesh independence study, $\mathbf{c}$ the computational mesh at the outlet boundary layer and the resolution of the particles
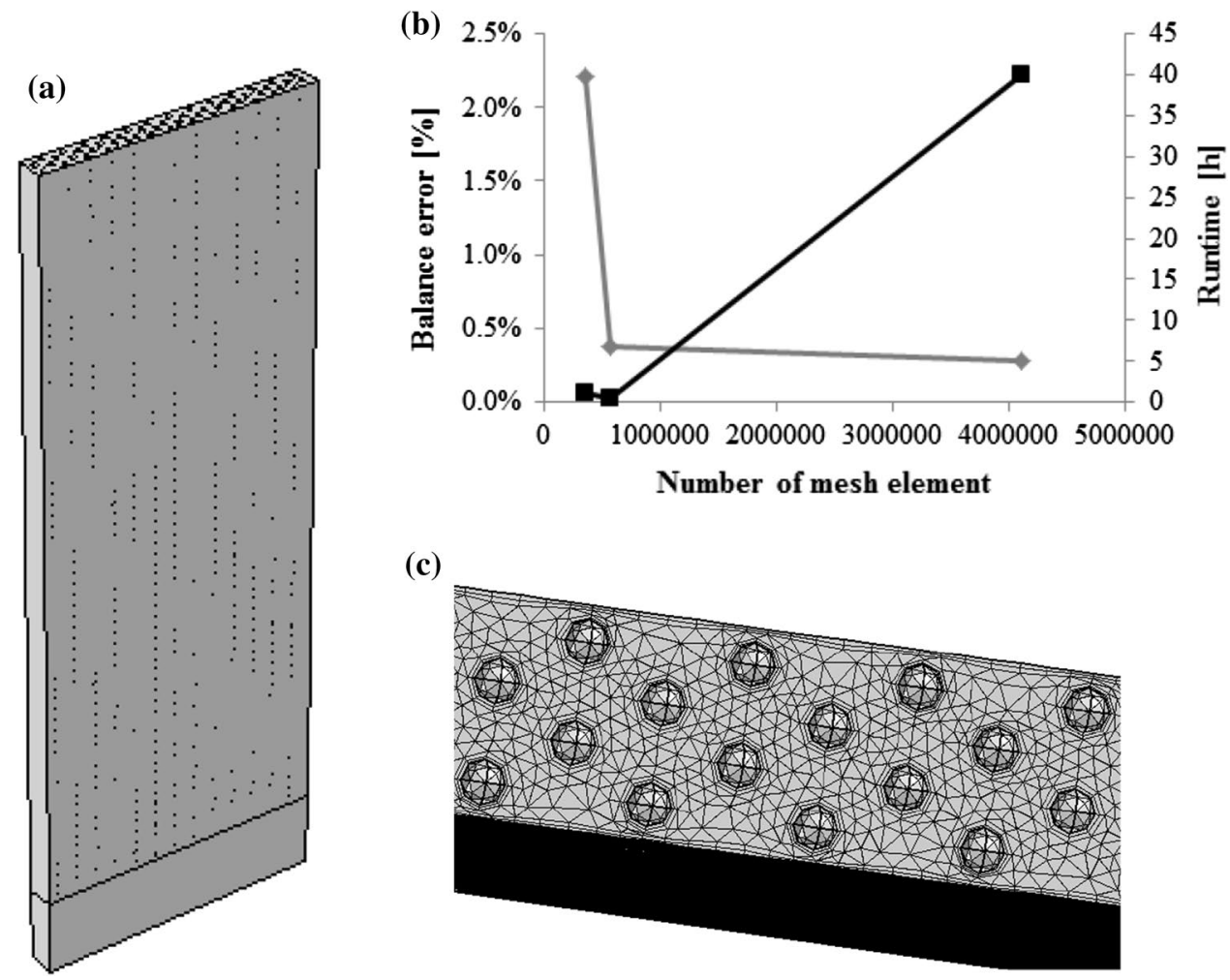

(c)

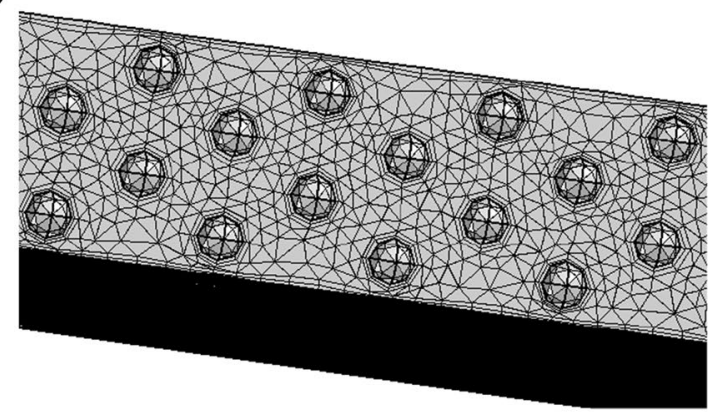

Plus software was used for the calculation of the heating of the gas and the compression work.

The laboratory-scale device is $1 \mathrm{~m}$ in height, $0.2 \mathrm{~m}$ in width and $0.02 \mathrm{~m}$ in depth. A temperature-dependent kinetic formula was applied to the particle scale models, and the boundary fluxes were calculated with different flow rates. The value of the boundary flux is determined by a $2 \mathrm{D}$ axisymmetric simulation which contains only one particle. The fluxes were then transferred to the larger-scale model, and only the upper part containing the majority of the particles was calculated. During the step of optimization, the RSM used the results of the simulation as input information. When the evaluation of the results was finished, a quadratic expression was fitted (Eq 1), and we calculated the optimal operational parameters for the VOC removal process.

$y=a+b \cdot T+c \cdot F+d \cdot T^{2}+e \cdot T \cdot F+f \cdot F^{2}$

In Eq $1 T$ is the scaled temperature, $F$ is the scaled flow rate, and $a-f$ are the coefficients.

Table 1 shows the experimental plan, the simulation experiments with different factors, and the outcomes (Outcome 1 is energy demand, while Outcome 2 is removal time). 

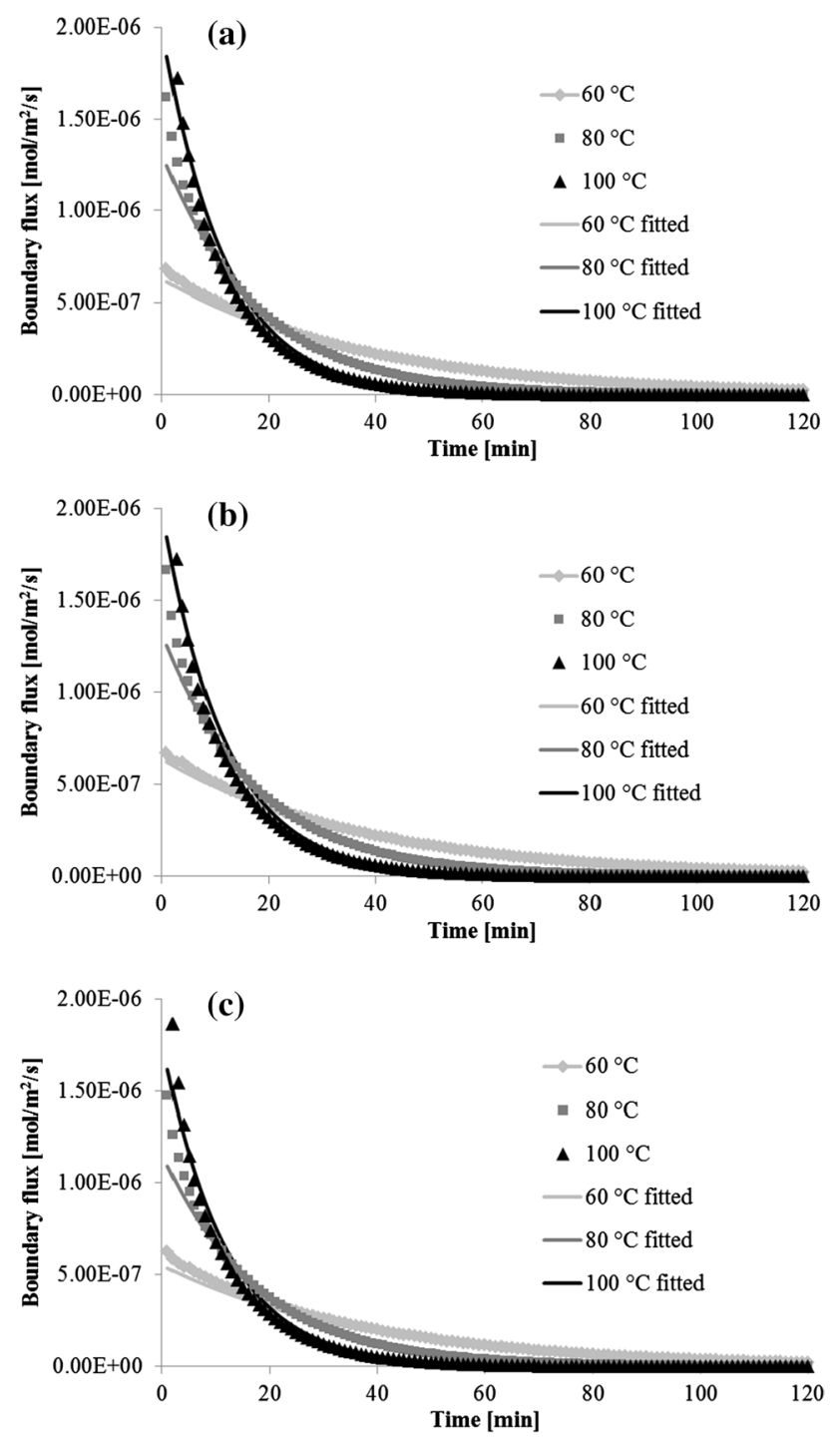

Fig. 3 The fitted curves for boundary fluxes a $3 \mathrm{~m}^{3} / \mathrm{h}, \mathbf{b} 6 \mathrm{~m}^{3} / \mathrm{h}$, and c $12 \mathrm{~m}^{3} / \mathrm{h}$

The economic optimum can be determined based on the simulation results. After the simulations have finished, we calculated a simplified environmental assessment for the system, evaluating the different cases with an LCA analysis. The energy usage and the environmental assessment were calculated based on a mix of primary energies representing the Hungarian primary energy usage (Fig. 1) (http://www.investhipa.hu/images/hipa_kiadvany_intro _greenenergy_web_2018).
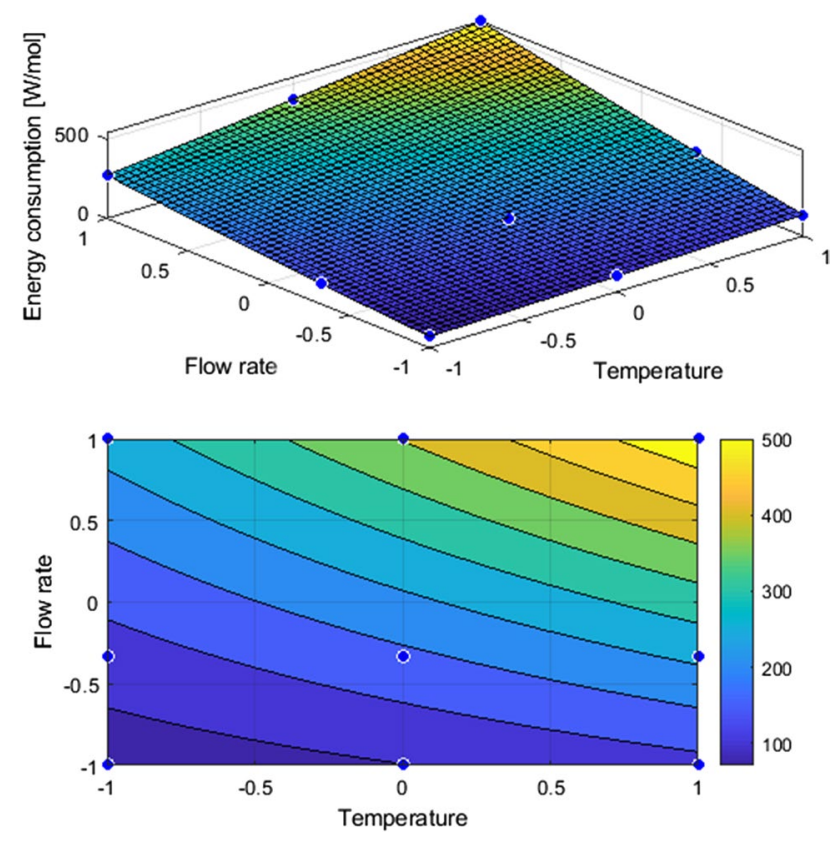

Fig. 4 The energy demand results $[\mathrm{W} / \mathrm{mol}]$
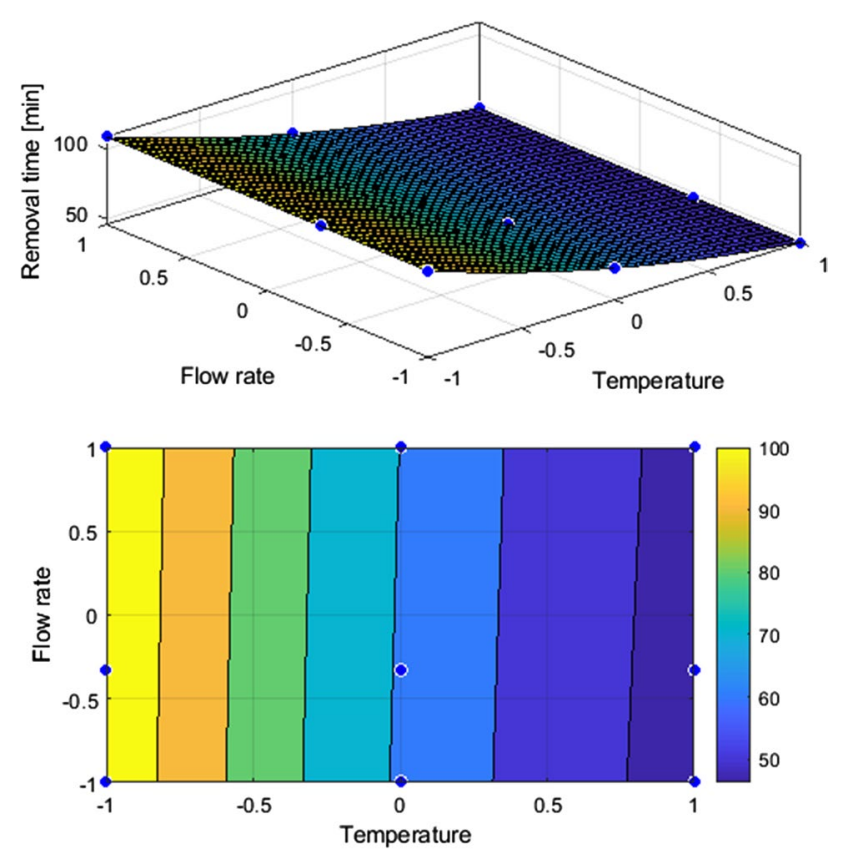

Fig. 5 The removal time results [s] 


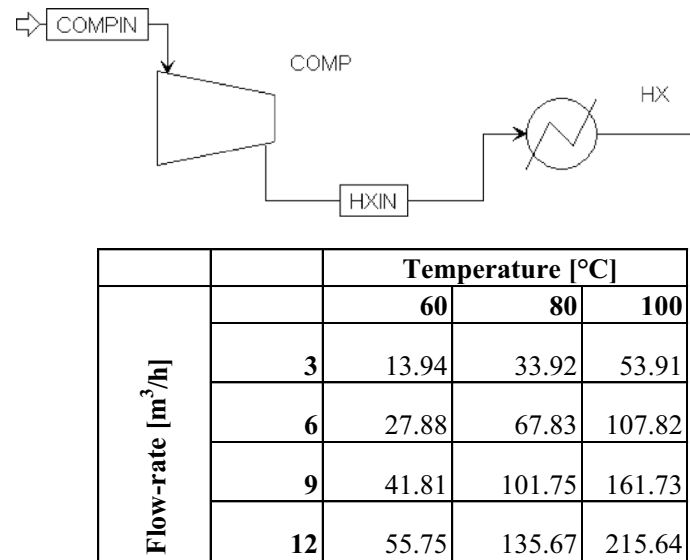

Fig. 6 The results of the simulation [kWs]

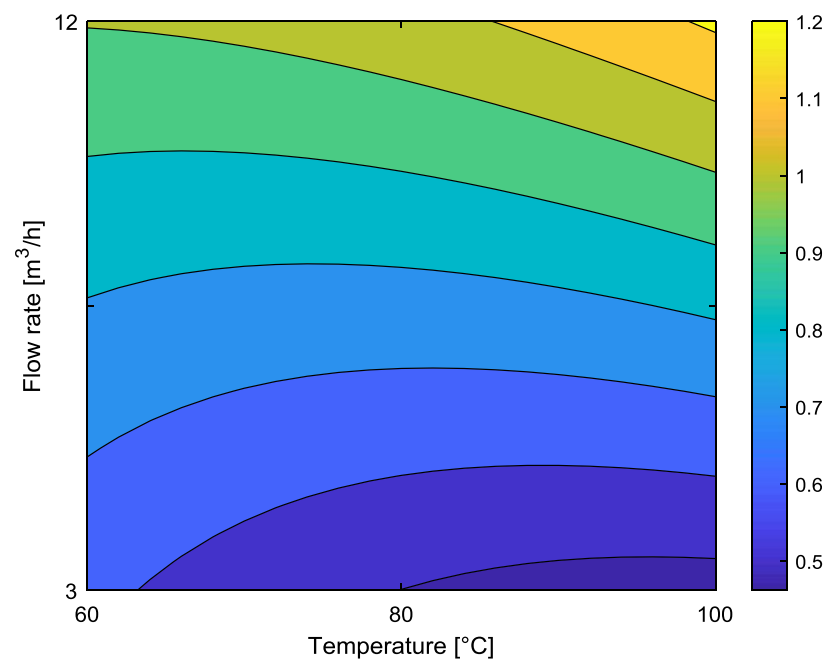

Fig. 7 Economic optimization plot

The most significant contributors in the energy mix are the oil and natural gas sectors that are causing most of the environmental impact. Two scenarios were defined in our LCA study. In the first case, the optimal operating point $\left(80{ }^{\circ} \mathrm{C}\right.$ and $3 \mathrm{~m}^{3} / \mathrm{h}$ nitrogen) was used, and the second was

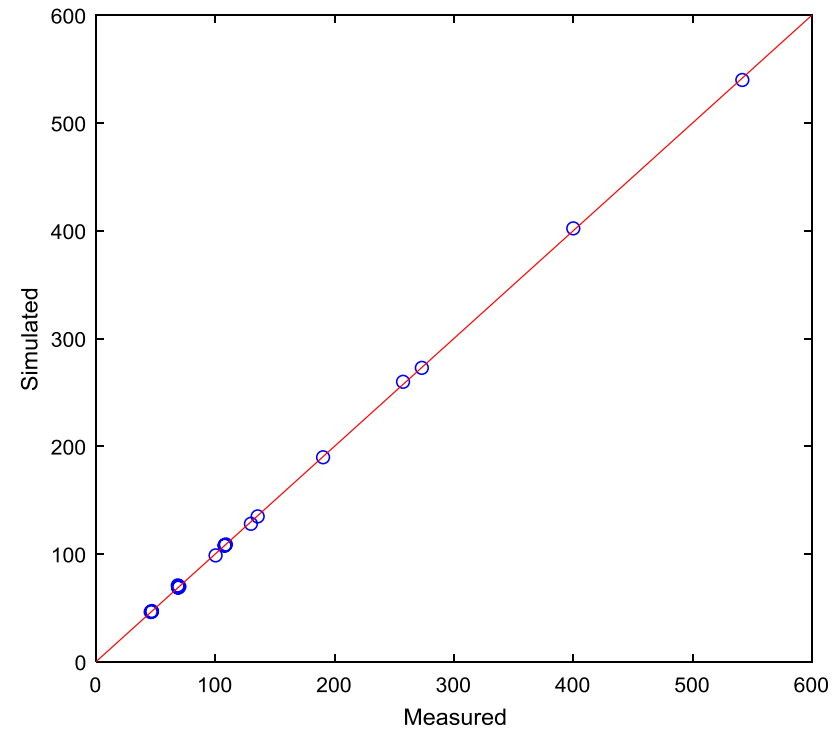

Fig. 8 Input data of the RSM model (measured-CFD) and the output results of the RSM model (simulated)

Table 2 The identified coefficients and significance levels

\begin{tabular}{lllll}
\hline Coefficient & $\begin{array}{l}\text { Outcome1 } \\
\text { (energy } \\
\text { demand) }\end{array}$ & $\begin{array}{l}\text { Significance } \\
\text { of outcome } \\
1\end{array}$ & $\begin{array}{l}\text { Outcome2 } \\
\text { (removal } \\
\text { time) }\end{array}$ & $\begin{array}{l}\text { Significance } \\
\text { of outcome 2 }\end{array}$ \\
\hline $\mathrm{a}$ & $2.4 \mathrm{E}+02$ & $2.2 \mathrm{E}-06$ & $6.9 \mathrm{E}+01$ & $2.0 \mathrm{E}-07$ \\
$\mathrm{~b}$ & $8.3 \mathrm{E}+01$ & $6.7 \mathrm{E}-06$ & $-3.1 \mathrm{E}+01$ & $2.9 \mathrm{E}-07$ \\
$\mathrm{c}$ & $1.5 \mathrm{E}+02$ & $1.1 \mathrm{E}-06$ & $5.0 \mathrm{E}-01$ & $4.8 \mathrm{E}-02$ \\
$\mathrm{~d}$ & $4.1 \mathrm{E}+00$ & $1.4 \mathrm{E}-01$ & $8.2 \mathrm{E}+00$ & $7.8 \mathrm{E}-05$ \\
$\mathrm{e}$ & $5.1 \mathrm{E}+01$ & $4.9 \mathrm{E}-05$ & $-5.4 \mathrm{E}-02$ & $7.9 \mathrm{E}-01$ \\
$\mathrm{f}$ & $1.1 \mathrm{E}+01$ & $1.7 \mathrm{E}-02$ & $1.9 \mathrm{E}-04$ & $1.0 \mathrm{E}+00$ \\
\hline
\end{tabular}

where the whole amount of VOC (defined as hexene in this case) was supposed to be emitted into the atmosphere.

The environmental problems were evaluated by the gateto-gate approach and TRACI2.1 methodology. In this case, it only took one process in account: the VOC removal. TRACI is the abbreviation of Tool for the Reduction and Assessment of Chemical and other environmental Impacts (Bare 2011). The system was implemented within OpenLCA software using the openlca_lcia_v2_0_4_20191024 (Oliva et al. 2018) database. According to the database, 2000 working hours were defined annually, which resulted 
Table 3 The results of scenarios (a) reference case with hexene emission (b) the case with VOC removal (optimal point)

\begin{tabular}{lll}
\hline a) & & \\
\hline Input & Output \\
\hline PP + VOC & Drying & PP \\
$4000 \mathrm{~kg}$ & & $3906 \mathrm{~kg}$ \\
& & Hexene \\
& & $94 \mathrm{~kg}$ \\
\hline (b) & \\
\hline Input & & Output \\
\hline PP + VOC & Purification & PP \\
$4000 \mathrm{~kg}$ & column & $3906 \mathrm{~kg}^{-}$ \\
Thermal energy & & Waste nitrogen \\
$67833 \mathrm{~W}$ & $6000 \mathrm{~m}^{3}$ \\
Power & & \\
$51994 \mathrm{~W}$ & & \\
Nitrogen & & \\
$6000 \mathrm{~m}^{3}$ & & \\
\hline
\end{tabular}

in approximately $4000 \mathrm{~kg}$ of purified plastic granulates. The amount of hexene emitted was calculated based on the amount of hexene left on the surface of the granulates. The treatment of waste nitrogen was not taken in consideration in this work. It can be purified using specific distillation processes. The LCA analysis provides the environmental optimum, which is always against the economic optimum. The best solution for a working system can be determined

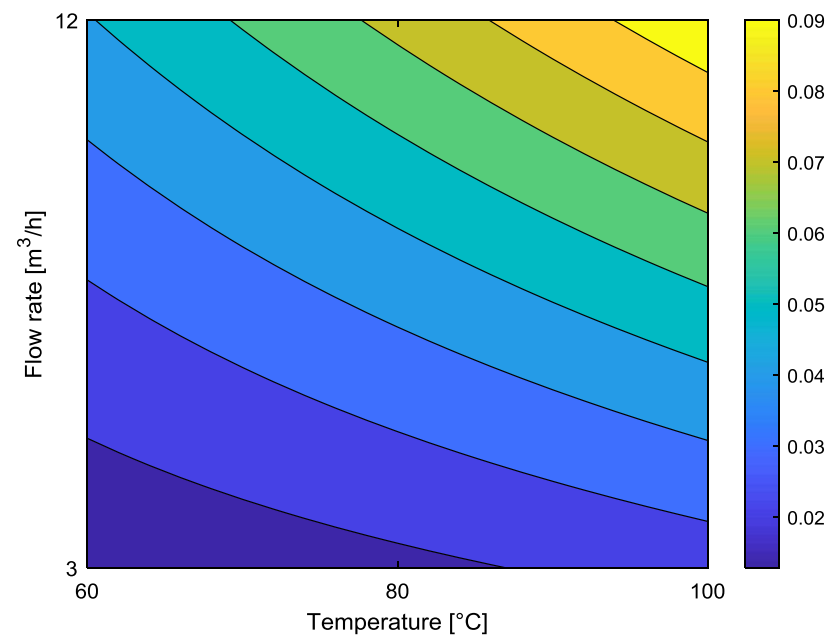

Fig. 9 The fossil fuel depletion map for the system by using an objective function that provides an optimum from both the economic and environmental points of view.

\section{Results and discussion}

This section shows the results of CFD modeling and the RSM. The detailed modeling of the purification column is performed using CFD modeling and RSM methodology. We defined the exact 3D geometry of the particles in the upper part of the column. Figure 2a shows the applied geometry.

When a multi-particle system is simulated, we have to compromise between the maximum feasible number of mesh element and the adequacy of the model. A mesh independence study was performed for the actual geometry, in which three different mesh sizes were applied. The balance error was calculated by the relative difference between the outlet and the inlet flow rates $\left(F_{\text {out }}-F_{\text {in }}\right) / F_{\text {in }}$. Figure $2 \mathrm{~b}$ shows the results of the mesh independence study, and Fig. 2c shows the resolution of the chosen (finest) mesh. The balance error is low enough for the second type of model ( $600 \mathrm{k}$ number). However, the mesh resolution in the particulate system is critical, and the mesh with the highest number of elements (around 4 million) resulted in a feasible runtime ( $\sim 0 \mathrm{~h}$ in an Intel Xeon E5620 computer with 72 GB RAM); therefore, it was chosen for the detailed simulation study. Figure 3 shows the boundary fluxes at three different flow

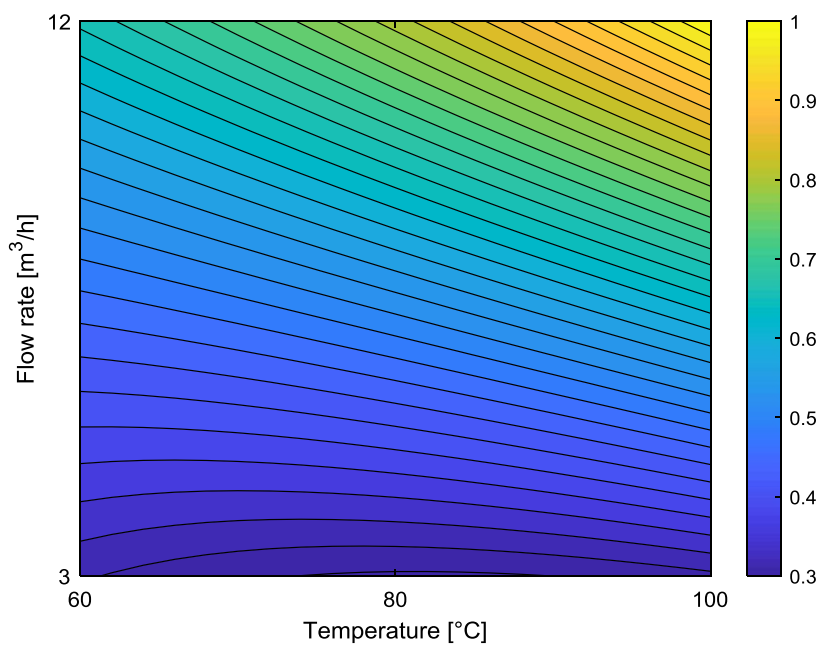

Fig. 10 The optimal operating intervals from both economical and environmental point of views 
rates: (a) $3 \mathrm{~m}^{3} / \mathrm{h}$, (b) $6 \mathrm{~m}^{3} / \mathrm{h}$, and (c) $12 \mathrm{~m}^{3} / \mathrm{h}$ ). Higher temperature leads to higher initial flux and a lower purification time as expected. The simulated values are represented by the markers, while the fitted curves are plotted as solid lines. An excellent agreement was obtained between the measurement and simulation. The resulted curves were implemented in the larger-scale model as boundary fluxes. As shown in Figs. 4 and 5, the optimization was performed using the RSM method.

The energy demand increases with the temperature and the flow rate (as it was expected), and the optimal value is found at the lowest values of factors. Aspen Plus v11 software was used for the calculation of energy demand using the compressor and heater models; the construction is represented in Fig. 6. Sensitivity analysis was applied to calculate the heat and compression demand. The higher temperature and flow rate will lead to higher power demand, and thus, a higher economic cost.

The removal time decreases significantly by increasing the temperature and slightly decreases with the flow rate; therefore, it appears to be more like an operational parameter.

The value of the removal time should be based on the technology that we have built for the elimination of VOC.

If the two factors are normalized and averaged, it results in Fig. 7, where the lowest values of removal time are shown at the lowest flow rates and higher temperatures. The operations with low environmental impact and removal time can be accomplished at this range of flow rate and temperature. Figure 8 shows the comparison of the input data of the RSM model (measured-CFD) and the output results of the RSM model (simulated). As displayed here, an excellent agreement was found between the two data sets.

Table 2 shows the identified coefficients and the significance of outcomes (based on probability ( $p$ ) values). The lower the significance value is, the higher impact it has on the outcome. In the case of temperature, the interaction term $(e)$ has the highest significance level, while all the terms containing $F$ (flow rate) are nonsignificant. The lowest temperature value appears to be the economically optimal value. The notation of the coefficients is the same as in Eq 1.

\section{Results of the LCA study}

Table 3 shows the inventory calculation of two scenarios, the reference case and the case with purification, where polypropylene is denoted by PP. The two cases resulted in two different types of environmental impacts. Case (a), emitting all of the VOC into the atmosphere resulted in 0.0153 CTUe level of ecotoxicity and contributed to the smog formation with a $516.28 \mathrm{~kg} \mathrm{O}_{3}$ equivalent. This case shows the unlikely scenario, where all the plastic granulates are left on open air to eliminate all the unnecessary hexene.

The VOC removal case (b) resulted in no environmental harm, except for fossil fuel depletion. Using the impact factors deriving from the database, we have created a fossil fuel depletion map for the process, which is shown in Fig. 9.

As the figure demonstrates, the minimal environmental impact can be observed in the lower parts of the graph. Although it conflicts with economic objectives, we found that the best operating point of the technology from the environmental point of view is the lower flow rate, and regarding temperature below $80{ }^{\circ} \mathrm{C}$. In the last part of our study, we took both the economic (Fig. 7) and the environmental (Fig. 9) objectives into consideration. The results are shown in Fig. 10. Based on that, we can conclude that respecting the two objectives, the optimal operation interval is at the medium temperature and lower nitrogen flow rate.

\section{Conclusion}

In this study, a detailed investigation of a VOC removal process was simulated using CFD simulation. A temperature-dependent kinetic was applied, which is close to the real industrial kinetics. The boundary fluxes calculated at the boundaries of the particles using the particle size model were transferred to the laboratory-scale model. RSM methodology was applied for the two-factor optimization of the system, using temperature and purge gas flow rate as factors. The two considered outcomes were the energy demand and removal time. The energy demand escalates with increase in the flow rate and the temperature. However, those two factors have a positive effect on the removal time as well. The RSM model concluded an 
economically optimal point, which was found in the range of lower flow rate and higher temperatures (from $80^{\circ} \mathrm{C}$ ).

A simplified LCA analysis was also performed comparing a reference case with the full emission and a purification scenario. OpenLCA was applied, and TRACI2.1 impact assessment was accomplished. The LCA resulted in an environmental optimum at temperatures below 80 ${ }^{\circ} \mathrm{C}$, and low flow rates.

Based on the environmental and economic optimization, the main conclusion of this study is that the optimal map concerns both the environmental and economic factors. Considering the colliding factors, an environmentally and economically feasible operating point was found at 80 ${ }^{\circ} \mathrm{C}$ of temperature and $3 \mathrm{~m}^{3} / \mathrm{h}$ of flow rate.

Acknowledgements Open access funding provided by University of Pannonia. The research was supported by EFOP-3.6.1-16-2016-00015 Smart Specialization Strategy (S3)-Comprehensive Institutional Development Program at the University of Pannonia to Promote Sensible Individual Education and Career Choices project. We would like to express our acknowledgement for the financial support of Széchenyi 2020 under the GINOP-2.2.1-15-2017-00059.

Open Access This article is licensed under a Creative Commons Attribution 4.0 International License, which permits use, sharing, adaptation, distribution and reproduction in any medium or format, as long as you give appropriate credit to the original author(s) and the source, provide a link to the Creative Commons licence, and indicate if changes were made. The images or other third party material in this article are included in the article's Creative Commons licence, unless indicated otherwise in a credit line to the material. If material is not included in the article's Creative Commons licence and your intended use is not permitted by statutory regulation or exceeds the permitted use, you will need to obtain permission directly from the copyright holder. To view a copy of this licence, visit http://creativecommons.org/licenses/by/4.0/.

\section{References}

Bare JC (2011) TRACI 2.0 - the tool for the reduction and assessment of chemical and other environmental impacts. Clean Technol Environ Policy 13(5):687-696
Berenjian A, Chan N, Malmiri HJ (2012) Volatile organic compounds removal methods: a review. Am J Biochem Biotechnol 8(4):220-229

Bobst R, Garner B, Jacob F (1983) Degassing process for removing unpolymerized monomers from olefin polymers. US Patent 4,372,758, (19)

Box GEP, Wilson KB (1951) On the experimental attainment of optimum conditions (with discussion). J R Stat Soc Ser B13(1):1-45

Coppedè A, Gaggero S, Vernengo G et al (2019) Hydrodynamic shape optimization by high fidelity CFD solver and Gaussian process based response surface method. Appl Ocean Res 90:101841

Costa D, Quinteiro P, Dias AC (2019) A systematic review of life cycle sustainability assessment: current state, methodological challenges, and implementation issues. Sci Total Environ 686:774-787

Dobslaw D, Schulz A, Helbich S et al (2017) VOC removal and odor abatement by a low-cost plasma enhanced biotrickling filter process. J Environ Chem Eng 5(6):5501-5511

Egedy A, Rado A (2018) CFD based optimization of a laboratory scale silo. In: COMSOL conference proceedings 2018

Egedy A, Gyurik L, Ulbert Z et al (2019) CFD modelling and simulation of a VOC removal silo. Chem Eng Trans 76:163-168

EPA-450 (1983) Control of volatile organic compound emissions for manufacture of high-density polyethylene, polypropylene, and polystyrene resins. US Environmental Protection Agency

Ghoreyshi AA, Sadeghifar H, Entezarion F (2014) Efficiency assessment of air stripping packed towers for removal of VOCs (volatile organic compounds) from industrial and drinking waters. Energy 73:838-843

Hemmati A, Rashidi H, Hemmati A et al (2019) Using rate based simulation, sensitivity analysis and response surface methodology for optimization of an industrial CO2 capture plant. J Nat Gas Sci Eng 62:101-112

http://www.investhipa.hu/images/hipa_kiadvany_intro_greenenerg y_web_201808.pdf, Accessed: 2019.12.16

Jung KA, Nam CW, Woo SH et al (2016) Response surface method for optimization of phenolic compounds production by lignin pyrolysis. J Anal Appl Pyrol 120:409-415

Oliva G, Zarra T, Naddeo V et al (2018) Comparative analysis of AOPs and biological processes for the control of VOCs industrial emissions. Chem Eng Trans 68:451-456

http://www.openlca.org/, Accessed: 2019.12.09

Shalmashi A, Khodadadi F (2019) Ultrasound-assisted synthesis of biodiesel from peanut oil by using response surface methodology. Energy Environ 30(2):272-291 
Song M, Liu X, Zhang Y et al (2019) Sources and abatement mechanisms of VOCs in southern China. Atmos Environ 201:28-40

Ström H, Sasic S, Thunman H (2015) Challenges and opportunities in the Eulerian approach to numerical simulations of fixed-bed combustion of biomass. Procedia Eng 102:1573-1582. https:// doi.org/10.1016/j.proeng.2015.01.293

Ubando AT, Rivera DRT, Chen WH et al (2019) A comprehensive review of life cycle assessment (LCA) of microalgal and lignocellulosic bioenergy products from thermochemical processes. Biores Technol 291:121837

Wang Q, Tang H, Ma Q et al (2019) Life cycle assessment and the willingness to pay of waste polyester recycling. J Clean Prod 234:275-284

Wehinger GD, Eppinger T, Kraume M (2015) Detailed numerical simulations of catalytic fixed-bed reactors: heterogeneous dry reforming of methane. Chem Eng Sci 122:197-209. https://doi. org/10.1016/j.ces.2014.09.007
White J (2012) CFD Simulation of silica gel and water adsorbent beds used in adsorption cooling system. Ph. D. Birmingham, 210

Xie R, Ji J, Guo K et al (2019) Wet scrubber coupled with UV/PMS process for efficient removal of gaseous VOCs: roles of sulfate and hydroxyl radicals. Chem Eng J 356:632-640

Yang Y, Liu X, Zheng J et al (2018) Characteristics of one-year observation of VOCs, NOx, and O3 at an urban site in Wuhan, China. J Environ Sci (China) 79:1-14

Zhou X, Duan Y, Huai X, Li X (2013) 3D CFD modeling of acetone hydrogenation in fixed bed reactor with spherical particles. Particuology 11(6):715-722. https://doi.org/10.1016/j.parti c.2012.10.009

Zoveidavianpoor M, Samsuri A, Shadizadeh S (2012) Health, safety, and environmental challenges of xylene in upstream petroleum industry. Energy Environ 23(8):1339-1352 